\title{
Clinical Study \\ The Influence of Self-Efficacy on Mood States in People with Spinal Cord Injury
}

\author{
Ashley Craig, ${ }^{1}$ Nirupama Wijesuriya, ${ }^{1}$ and Yvonne Tran ${ }^{1,2}$ \\ ${ }^{1}$ Rehabilitation Studies Unit, Sydney Medical School-Northern, The University of Sydney, P.O. Box 6, Ryde, \\ Sydney, NSW 1680, Australia \\ ${ }^{2}$ Key University Centre for Health Technologies, University of Technology, Broadway, Sydney, NSW 2007, Australia
}

Correspondence should be addressed to Ashley Craig; a.craig@sydney.edu.au

Received 18 January 2013; Accepted 17 February 2013

Academic Editors: P. Czarnecki, J. D. Kingsley, J. J. Sosnoff, and M. Yu

Copyright (C) 2013 Ashley Craig et al. This is an open access article distributed under the Creative Commons Attribution License, which permits unrestricted use, distribution, and reproduction in any medium, provided the original work is properly cited.

\begin{abstract}
Objective. Negative mood is prevalent in people with a neurological injury such as spinal cord injury (SCI). However, research is needed for determining those people with SCI who are vulnerable to negative mood states, as well as establishing the influence of self-efficacy, that is, expectations of their control over their lives. The objective of this research was to investigate the protective role that self-efficacy may play in adult people with SCI compared to able-bodied controls. Methods. Participants included 41 adults with SCI living in the community and 41 able-bodied controls similar in age, sex ratio, and education. All participants completed a psychological assessment regimen in a relaxed environment. Measures consisted of validated measures of self-efficacy and negative mood states. Results. The SCI group was found to have significantly elevated levels of depressive mood, anxiety, stress, and poor self-efficacy. SCI participants with low levels of self-efficacy were shown to have significantly elevated levels of depressive mood and anxiety in comparison to those SCI participants with high levels of self-efficacy and able-bodied controls. Conclusions. People with a neurological injury such as SCI are vulnerable to experiencing clinically elevated negative mood states if they have poor expectations of control over their lives. Implications for SCI rehabilitation are discussed.
\end{abstract}

\section{Introduction}

Spinal cord injury (SCI) is a chronic neurological disorder that involves the cord being severely bruised, lacerated, or severed during a traumatic injury or damaged as a result of disease. The risk of suffering an SCI is low; however, when it occurs it is usually very traumatic and debilitating with substantial negative impacts on quality of life (QOL) $[1,2]$. Most injuries to the spinal cord in adults involve damage to the surrounding protective vertebral column, consisting of the cervical, thoracic, lumbar, sacral, and the coccygeal vertebrae. Neurological damage resulting from an SCI depends upon the degree to which the injury disturbs or intrudes into the spinal cord [1]. Extreme damage to the spinal cord leads to loss of sensation and paralysis of voluntary muscles, resulting in reduced mobility and independence in activities of daily living and impairment of social and vocational skills. Potential negative influences on respiratory, cardiovascular, urinary, gastrointestinal, and reproductive systems also occur
[1]. Furthermore, chronic pain, chronic fatigue, and mental health dysfunction are common secondary conditions that contribute to decreased QOL [3-10]. Mental health dysfunction in the form of negative mood states such as depression is a risk for up to $40 \%$ of adults with SCI living in the community [7]. This high prevalence rate highlights the need for careful screening of SCI patients, especially as they come to the end of their inpatient rehabilitation and are about to be discharged into the community [5]. Arguably, if those at risk of depression can be offered early treatment, then adjustment to SCI would improve substantially.

Outcomes like restrictions in mobility, barriers to employment and social engagement, and a high dependence on caregivers will combine to challenge expectations of control (called self-efficacy) in people with SCI [7]. Among other goals, SCI rehabilitation should enhance feelings of selfmanagement (or self-efficacy) by teaching skills that convince the person they can control and reduce the negative impacts of associated impairment, as well as lower social barriers 
by improving locomotor function and enhancing vocational capacity [2]. Self-efficacy and expectations of control [11] have been shown to be important factors influencing health in diverse areas including weight loss [12], improvement following stroke [13], and improvement in stuttering [14] and in SCI rehabilitation outcomes [15-18]. Self-efficacy has been defined as a person's belief or sense of confidence in his or her own ability to perform a particular task or behavior successfully in the future [11]. Horn and colleagues found significant associations between low self-efficacy and level of neurological impairment [16]. Craig and colleagues found an association between low self-efficacy in people with SCI with long-term risks of depressive mood and poor outcomes in rehabilitation $[17,18]$.

Controlled research investigating the association of selfefficacy with negative mood states like depressive mood and anxiety in adult people with SCI is scarce. Therefore, the major objective of this research was to investigate more carefully the association between negative mood states and selfefficacy in adult people with SCI and able-bodied controls. To achieve this goal, differences in negative mood states were compared with adult people with SCI with low versus high self-efficacy, as well as with able-bodied controls. It was hypothesized that higher levels of negative mood states would occur in people with SCI with low self-efficacy compared to people with high self-efficacy with or without SCI.

\section{Materials and Methods}

2.1. Study Participants. Participants included 41 adults with SCI living in the community and 41 able-bodied controls. The SCI participants were enrolled into the study from rehabilitation units as well as through advertising in disability newsletters. The controls were enrolled by advertising and were admitted into the study if they stated they were free of chronic physical or psychiatric disease at the time of assessment. The 41 controls were selected and matched to the SCI participants for sex and age ( \pm 5 years) on a case by case basis from a sample of around 100 able-bodied participants who, along with the SCI participants, were enrolled in a neuropsychophysiological study.

The mean age for the SCI group was 47.1 years $(\mathrm{SD}=12$; $\min =21, \max =70)$ consisting of 39 males and 2 females. The mean age for the controls was 46.8 years $(S D=12$; $\min =21, \max =70$ ) also consisting of 39 males and 2 females. The two females in each group were aged in their mid 50 s to early 60 s. The two females were left in the study as the statistical outcomes with the two removed did not change. Their inclusion increases the statistical power of the study. Twenty-seven SCI participants had paraplegia, 14 had tetraplegia, while 23 were assessed by a medical specialist to have an incomplete lesion with the remaining 18 having complete lesions. The mean time since the injury was 16.5 years $(\mathrm{SD}=14)$. The majority $(n=23)$ sustained an SCI in a road accident, 9 by a fall, 6 in sporting injury, and 3 due to a medical condition. The SCI and control groups had similar years of education (15.9 years, $\mathrm{SD}=2.0 ; 14$ years, $\mathrm{SD}=2.6$, resp.). Most of the SCI participants $(n=24)$ were on medications, the main types taken being analgesics, antispasmodics, hypnotics, and antidepressants. Most $(n=$ 32) of the SCI sample did not have a comorbid TBI.

Research ethics approval was received from the local institutional human research ethics committee, and written consent was obtained prior to participation in the study. Inclusion criteria for SCI participants consisted of (a) the presence of a chronic SCI, (b) age 20 or more and less than 75 years old at the time of interview, and (c) ability to speak and understand instructions in English. Inclusion criteria for the controls were the same for points (b) and (c). Exclusion criteria for the controls included the presence of a chronic neurological injury.

2.2. Psychological Measures. Psychological questionnaires were completed in a relaxed environment in either the participants' home or in a clinic. All participants completed the Depression Anxiety Stress Scales (DASS), a self-report questionnaire assessing anxiety, depressive mood, and stress [19]. High scores indicate elevated mood states. The DASS has acceptable reliability and discriminate validity [20]. They also completed the Lifestyle Appraisal Questionnaire (LAQ) which is a standardized two-part self-report Likert measure of cumulative health risks (LAQ1) and self-efficacy (LAQ2) [21]. Only LAQ2 data is presented in this paper. The LAQ2 measures a person's self-efficacy, specifically, their perceived ability to influence life challenges. High scores indicate low self-efficacy. The LAQ2 has acceptable test-retest reliability and internal reliability (Cronbach's coefficient alpha of 0.89 ), as well as acceptable construct validity and low social desirability [21].

2.3. Data Analysis. Differences between groups for the psychological measures were determined by first conducting MANOVA, followed by one-way ANOVA analyses to determine where differences occurred. The influence of self-efficacy on negative mood states was determined by dichotomizing the groups into low and high self-efficacy subgroups on the basis of the population mean LAQ2 score of 20 [21]. Scores lower than 20 indicate enhanced self-efficacy, while scores equal to or over 20 indicate poor self-efficacy [21]. The DASS depressive mood, anxiety, and stress scores were then calculated for the SCI and able-bodied participants who were then placed into low versus high self-efficacy subgroups. Those participants with self-efficacy scores less than 20 constituted the high self-efficacy subgroups, while scores equal to or above 20 constituted the low self-efficacy subgroups in the SCI and control groups. MANOVA was performed first to determine whether differences occurred between the three mood state measures for the four subgroups. This was followed by a series of factorial ANOVAs so as to determine whether interaction effects were significant for differences in negative mood states as a function of high versus low self-efficacy. Bonferroni protected $t$-tests were then used to determine where differences occurred. Etasquared $\left(\eta^{2}\right)$ values are provided for all analyses as an indication of the size of the difference between the two samples. An $\eta^{2}$ of around .03 is considered small, .13 is considered 
TABLE 1: Negative mood states (DASS) and self-efficacy (LAQ2) scores for the SCI group $(n=41)$ and the able-bodied controls $(n=41)$. ANOVA statistics, probabilities of difference, and effect sizes $\left(\eta^{2}\right)$ are shown.

\begin{tabular}{|c|c|c|c|c|}
\hline Variable & SCI group mean (SD) 95\% CI & Able-bodied controls mean (SD) 95\% CI & F & $\eta^{2}$ \\
\hline Dep. mood & $9.5(11.1) 6.0-13.0$ & $2.9(3.6) 1.7-4.0$ & $13.0^{* *}$ & 0.14 \\
\hline Anxiety & $5.3(7.7) 2.9-7.8$ & $2.6(2.9) 1.7-3.6$ & $4.2^{*}$ & 0.05 \\
\hline Stress & $10.5(8.7) 7.7-13.3$ & $7.3(4.8) 5.8-8.8$ & $4.3^{*}$ & 0.05 \\
\hline LAQ2 & 19.8 (16.1) $14.7-24.9$ & $14.1(7.3) 11.7-16.4$ & $4.2^{*}$ & 0.05 \\
\hline
\end{tabular}

${ }^{*} P<.05,{ }^{* *} P<.01$.

TABLE 2: DASS depressive mood, anxiety, and stress domain scores across low versus high levels of self-efficacy.

\begin{tabular}{lcccc}
\hline \multirow{2}{*}{ DASS mood states } & \multicolumn{2}{c}{ Low self-efficacy $\geq 20$ on the LAQ2 } & \multicolumn{2}{c}{ High self-efficacy <20 on the LAQ2 } \\
& SCI mean (SD) & Controls mean (SD) & SCI mean (SD) & Controls mean (SD) \\
\hline Dep. mood & $20.5(10.9)$ & $6.2(4.5)$ & $3.1(3.9)$ & $2.3(3.1)$ \\
Anxiety & $10.7(10.5)$ & $4.0(1.8)$ & $2.2(2.5)$ & $2.4(3.0)$ \\
Stress & $17.6(9.1)$ & $12.0(4.8)$ & $6.4(5.3)$ & $6.5(4.4)$ \\
\hline
\end{tabular}

There were 15 in the low self-efficacy SCI subgroup and 26 in the high self-efficacy SCI subgroup. There were 6 in the low self-efficacy able-bodied control group and 35 in the high self-efficacy subgroup.

a medium difference, and over .2 is considered a large and substantial difference [22]. Post hoc statistical power of the test is also provided. All analyses were performed using Statistica Software (Version 9, StatSoft).

\section{Results}

Age, sex, and years of education were not found to influence significantly the level of mood states. Table 1 shows DASS domains depressive mood, anxiety, and stress scale scores, as well as LAQ2 self-efficacy scores for the two groups, including ANOVA results and effect sizes $\left(\eta^{2}\right)$. The SCI group was found to be significantly different from the able-bodied control group for the three DASS mood state domains and for the LAQ2 self-efficacy measure: Wilks $\lambda=.85, F_{4,77}=3.5, P<$ $.05, \eta^{2}=.16$, power $=85 \%$. Table 1 also shows the ANOVA results for differences between groups for each individual domain. Inspection of Table 1 shows that the overall SCI group had significantly higher levels of depressive mood, anxiety, stress, and poorer self-efficacy when compared to the able bodied group.

3.1. The Impact of Self-Efficacy on Mood States. After dichotomizing into the self-efficacy subgroups, the mean LAQ2 scores for the high self-efficacy SCI and able-bodied subgroups were 10.2 (SD = 5.4; 95\% CI: 8.0-12.4) and $11.9(\mathrm{SD}=$ 4.7; 95\% CI: 10.3-13.5), respectively. The mean LAQ2 scores for the low self-efficacy SCI and able-bodied subgroups were $36.4(\mathrm{SD}=14.9 ; 95 \% \mathrm{CI}: 28.1-44.7)$ and $26.5(\mathrm{SD}=6.8 ; 95 \%$ CI: 19.3-33.7), respectively. Table 2 shows DASS depressive mood, anxiety, and stress domain scores as a function of low versus high levels of self-efficacy for the SCI ( $n=15$ with low self-efficacy and $n=26$ with high self-efficacy) and control groups ( $n=6$ with low self-efficacy and 35 with high self-efficacy). MANOVA indicated that significant effects occurred for group: $F_{3,76}=8.3, P<.001, \eta^{2}=.25$, power $=99 \%$; for level of self-efficacy: $F_{3,76}=16.5, P<.0001$, $\eta^{2}=.39$, power $=99 \%$; and for the interaction effect: $F_{3,76}=$ $6.8, P<.001, \eta^{2}=.21$, power $=97 \%$.

Factorial ANOVA results indicated that a significant interaction effect occurred for depressive mood as a function of low versus high self-efficacy across the two groups: $F_{1,78}=$ 18.8, $P<.001, \eta^{2}=.19$, power $=99 \%$. Post hoc Bonferroni protected $t$-tests confirmed that the low self-efficacy SCI subgroup had significantly elevated depressive mood compared to the high self-efficacy SCI subgroup and the two able-bodied subgroups $(P<.01)$. Factorial ANOVA indicated that a significant interaction effect also occurred for anxiety as a function of low versus high self-efficacy across the two groups: $F_{1,78}=6.1, P<.01, \eta^{2}=.07$, power $=68 \%$. Post hoc Bonferroni protected $t$-tests confirmed that the low self-efficacy SCI subgroup had significantly elevated anxiety compared to the high self-efficacy SCI subgroup and the two able-bodied subgroups $(P<.05)$. The interaction effect for stress was close to significance $(P<.07)$. Figure 1 illustrates the interaction effects for self-efficacy on the depressive mood, anxiety, and Stress domains in the four subgroups.

\section{Discussion}

Unsurprisingly, the distress and upset associated with sustaining a severe neurological injury like SCI can be extreme. For instance, as stated previously, evidence suggests that up to $40 \%$ of adults with SCI will be at risk of developing a mental health disorder such as depression [7], about 56\% will develop excessive levels of chronic fatigue $[8,9]$, and up to $70 \%$ will develop chronic pain [23]. Interestingly, based on DASS norms [19], Table 1 shows that the SCI group as a whole had a depressive mood score at the bottom of the mild depressive mood range. The SCI group anxiety score was in the normal range as was the stress score. This suggests that as a group, the participants with SCI are adjusting well enough. However, there is clearly a proportion of participants with SCI who are scoring at least into the moderate range 
Wilks $\lambda=0.78864, F(3,76)=6.7895, P=0.0041$ vertical bars denote 0.95 confidence intervals

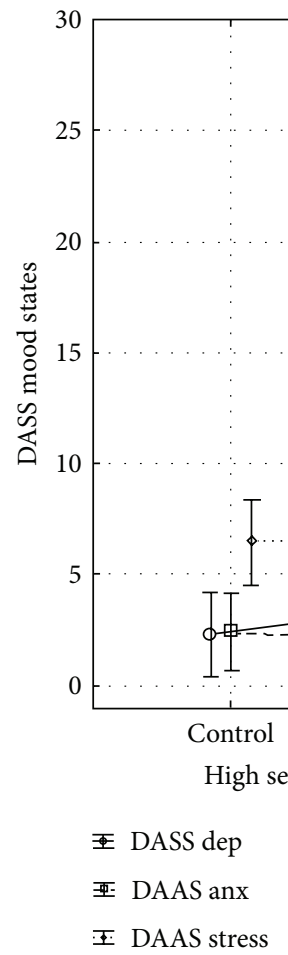

(a)

Wilks $\lambda=0.78864, F(3,76)=6.7895, P=0.0041$ vertical bars denote 0.95 confidence intervals

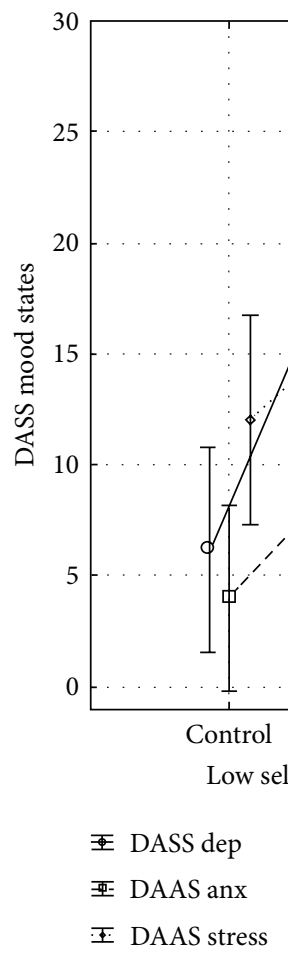

(b)

FIGURE 1: The influence of self-efficacy on negative mood states in adult people with SCI and able-bodied controls. for depressive mood and anxiety, given the standard deviations scores shown in Table 1 . Given the previously mentioned risk of chronic secondary conditions and consequent risks of poor adjustment when coping with the impairment and pain associated with SCI, rehabilitation strategies are needed that can protect people from the constant distress associated with SCI. The findings presented in this paper provide some direction in this regard, that is, the important role played by self-efficacy as a possible protective factor in people suffering an SCI.

Poor self-efficacy in adult people with SCI was found to be highly associated with elevated levels of depressive mood and anxiety. The low self-efficacy SCI subgroup had significantly elevated depressive mood and anxiety compared to SCI participants with high self-efficacy and to the ablebodied controls. Based on DASS norms [19], Table 2 shows that the low self-efficacy SCI subgroup had a Depressive mood score in the moderate to severe range, an Anxiety score in the moderate range, and Stress score in the mild range. Conversely, the high self-efficacy SCI subgroup had mood state scores in the normal DASS range [19], similar to the ablebodied group scores regardless of self-efficacy (though there was a trend for the low self-efficacy able-bodied subgroup to have higher levels of negative mood states than the high selfefficacy subgroup).

Self-efficacy has been established as a protective factor against negative mood in prior studies [24-27]. It has been suggested that it mediates between adverse life events and adjustment in people with SCI [7]. For example, Maciejewski and colleagues [27] showed that self-efficacy was a significant predictor of depressive symptom severity. They showed that having a higher level of self-efficacy resulted in fewer depressive mood symptoms in adults and that self-efficacy mediated around $40 \%$ of the effects of stressful life events on depressive mood. They concluded that maintaining a healthy self-efficacy, that is, a strong sense of control over one's life and environment, serves to protect a person from psychopathology, perhaps by ameliorating the negative effect of stressful life events [27]. Rutter [24] also argued that stressful life events may serve to precipitate psychopathology and that selfefficacy can act as a protective factor against the negative influence of stressful life events. Arnstein and colleagues showed that chronic pain was mediated by self-efficacy [26] and concluded that the belief in one's ability to cope with pain and disability protects against depression and anxiety. Middleton et al. [2] conducted research with over 100 adult persons with SCI living in the community and found that low self-efficacy was associated with substantially reduced QOL, above and beyond the effect of any physical impairment of the SCI. While the findings presented in this paper cannot conclude that self-efficacy plays a mediator role, the strong relationship found, coupled with prior research, does suggest that self-efficacy protects against the development of negative mood symptoms in people with SCI.

There are limitations in this study that require discussion. Participant numbers in the two groups were moderate, resulting in lowered statistical power [22], and dichotomizing the samples into self-efficacy subgroups would have lowered statistical power further. However, effect sizes for group 
differences were shown to be moderate to large, and post hoc power estimates confirmed that the statistical power in the study was robust, ensuring that the risk of a Type II error rate was low [22]. Furthermore, to clarify the mediator role of self-efficacy as a protective agent, research is now required that employs a longitudinal design in which participants are followed and measured over time, providing the necessary data to establish whether self-efficacy is indeed a mediator of stress or just a significant correlate.

\section{Conclusions}

Important clinical implications arise for improving the life of people with SCI. As argued before, problems like sadness related to their injury and impairment, chronic pain, and chronic fatigue have been reported by patients with SCI as a major source of distress and disablement $[4,7,9]$ which can lead to social isolation, anxiety, depression, and reduced QOL $[2,5]$. On the positive side, many people with SCI are coping satisfactorily, gaining employment, and socially integrating into their communities [28]. Therefore, clever assessment strategies are required during SCI rehabilitation that can detect in a reliable manner those who are at risk of maladaptive adjustment. Poor levels of self-efficacy may well be a primary risk factor for maladaptive adjustment. That is, those people with SCI who have no or little expectation of managing their lives and daily functions may well be those who are most at risk of depression, social withdrawal, pain, and so on. Validated self-efficacy measures are required to detect those at risk, and there are such measures available that could be used for this purpose $[15,21]$. Furthermore, there is a crucial need for studies that evaluate the efficacy of psychological-based rehabilitation strategies that can improve and enhance the self-efficacy of patients with SCI when in hospital and when living in the community [18, 25]. This remains a challenge for future research in the area.

\section{Acknowledgments}

The authors acknowledge the support of the New South Wales Premier SCI Grant (RIPG4) and by a Grant from the Australian Research Council (LP0560590).

\section{References}

[1] M. F. Sommer, Spinal Cord Injury. Functional Rehabilitation, Prentice Hall, New Jersey, NJ, USA, 2001.

[2] J. Middleton, Y. Tran, and A. Craig, "Relationship between quality of life and self-efficacy in persons with spinal cord injuries," Archives of Physical Medicine and Rehabilitation, vol. 88, no. 12, pp. 1643-1648, 2007.

[3] J. Middleton and A. Craig, "Psychological challenges in treating persons with spinal cord injury," in Psychological Dynamics Associated with Spinal Cord Injury Rehabilitation: New Directions and Best Evidence, A. Craig and Y. Tran, Eds., Nova Science, New York, NY, USA, 2008.

[4] N. Westgren and R. Levi, "Quality of life and traumatic spinal cord injury," Archives of Physical Medicine and Rehabilitation, vol. 79, no. 11, pp. 1433-1439, 1998.
[5] C. H. Bombardier, J. S. Richards, J. S. Krause, D. Tulsky, and D. G. Tate, "Symptoms of major depression in people with spinal cord injury: implications for screening," Archives of Physical Medicine and Rehabilitation, vol. 85, no. 11, pp. 1749-1756, 2004.

[6] M. J. Haran, B. B. Lee, M. T. King, O. Marial, and M. R. Stockler, "Health status rated with the medical outcomes study 36-item short-form health survey after spinal cord injury," Archives of Physical Medicine and Rehabilitation, vol. 86, no. 12, pp. 22902295, 2005.

[7] A. Craig, Y. Tran, and J. Middleton, "Psychological morbidity and spinal cord injury: a systematic review," Spinal Cord, vol. 47, no. 2, pp. 108-114, 2009.

[8] N. Wijesuriya, Y. Tran, J. Middleton, and A. Craig, "The impact of fatigue on the health related quality of life in persons with spinal cord injury," Archives Physical Medicine Rehabilitation, vol. 93, pp. 319-324, 2012.

[9] A. Craig, Y. Tran, N. Wijesuriya, and J. Middleton, "Fatigue and tiredness in people with spinal cord injury," Journal of Psychosomatic Research, vol. 73, pp. 205-210, 2012.

[10] M. P. Jensen, M. J. Chodroff, and R. H. Dworkin, "The impact of neuropathic pain on health-related quality of life: review and implications," Neurology, vol. 68, no. 15, pp. 1178-1182, 2007.

[11] A. Bandura, "Self-efficacy: toward a unifying theory of behavioral change," Psychological Review, vol. 84, no. 2, pp. 191-215, 1977.

[12] P. D. Martin, G. R. Dutton, and P. J. Brantley, "Self-efficacy as a predictor of weight change in African-American women," Obesity Research, vol. 12, no. 4, pp. 646-651, 2004.

[13] G. Robinson-Smith, M. V. Johnston, and J. Allen, "Self-care selfefficacy, quality of life, and depression after stroke," Archives of Physical Medicine and Rehabilitation, vol. 81, no. 4, pp. 460-464, 2000.

[14] G. Andrews and A. Craig, "Prediction of outcome after treatment for stuttering," British Journal of Psychiatry, vol. 153, pp. 236-240, 1988.

[15] J. W. Middleton, R. L. Tate, and T. J. Geraghty, "Self-efficacy and spinal cord injury: psychometric properties of a new scale," Rehabilitation Psychology, vol. 48, no. 4, pp. 281-288, 2003.

[16] W. Horn, W. Yoels, D. Wallace, D. Macrina, and M. Wrigley, "Determinants of self-efficacy among persons with spinal cord injuries," Disability and Rehabilitation, vol. 20, no. 4, pp. 138-141, 1998.

[17] A. R. Craig, K. M. Hancock, and H. G. Dickson, "Spinal cord injury: a search for determinants of depression two years after the event," British Journal of Clinical Psychology, vol. 33, no. 2, pp. 221-230, 1994.

[18] A. R. Craig, K. Hancock, and E. Chang, "The influence of spinal cord injury on coping styles and self-perceptions two years after the injury," Australian and New Zealand Journal of Psychiatry, vol. 28, no. 2, pp. 307-312, 1994.

[19] S. H. Lovibond and P. F. Lovibond, Manual for the Depression Anxiety Stress Scales, Psychology Foundation, Sydney, Australia, 1995.

[20] J. D. Henry and J. R. Crawford, "The short-form version of the depression anxiety stress scales (DASS-21): construct validity and normative data in a large non-clinical sample," British Journal of Clinical Psychology, vol. 44, no. 2, pp. 227-239, 2005.

[21] A. Craig, K. Hancock, and M. Craig, "The lifestyle appraisal questionnaire: a comprehensive assessment of health and stress," Psychology and Health, vol. 11, no. 3, pp. 331-343, 1996. 
[22] J. Cohen, Statistical Power Analysis for the Behavioral Sciences, LEA, New Jersey, NJ, USA, 1988.

[23] P. J. Siddall, J. M. McClelland, S. B. Rutkowski, and M. J. Cousins, "A longitudinal study of the prevalence and characteristics of pain in the first 5 years following spinal cord injury," Pain, vol. 103, no. 3, pp. 249-257, 2003.

[24] M. Rutter, "Resilience in the face of adversity: protective factors and resistance to psychiatric disorder," British Journal of Psychiatry, vol. 147, pp. 598-611, 1985.

[25] A. Craig, "Resilience in people with physical disabilities," in The Oxford Handbook of Rehabilitation Psychology, P. Kennedy, Ed., chapter 26, Oxford University Press, Oxford, UK, 2012.

[26] P. Arnstein, M. Caudill, C. L. Mandle, A. Norris, and R. Beasley, "Self efficacy as a mediator of the relationship between pain intensity, disability and depression in chronic pain patients," Pain, vol. 80, no. 3, pp. 483-491, 1999.

[27] P. K. Maciejewski, H. G. Prigerson, and C. M. Mazure, "Self-efficacy as a mediator between stressful life events and depressive symptoms. Differences based on history of prior depression," British Journal of Psychiatry, vol. 176, pp. 373-378, 2000.

[28] A. Craig, K. Hancock, and H. Dickson, "Improving the longterm adjustment of spinal cord injured persons," Spinal Cord, vol. 37, no. 5, pp. 345-350, 1999. 


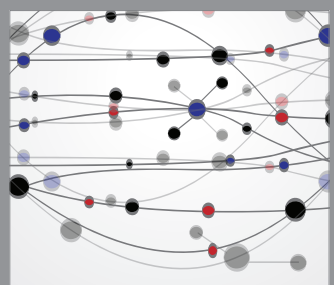

The Scientific World Journal
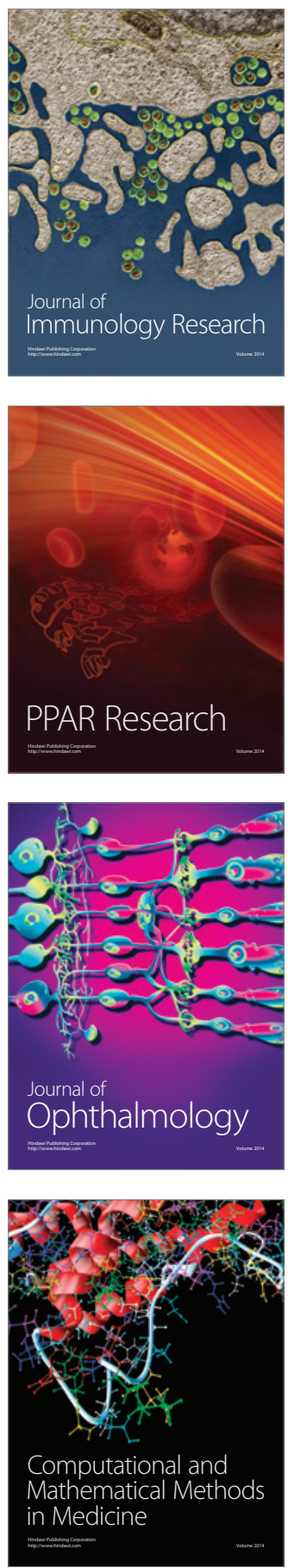

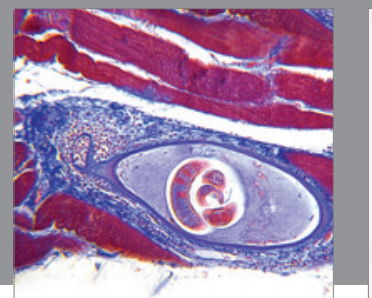

Gastroenterology

Research and Practice
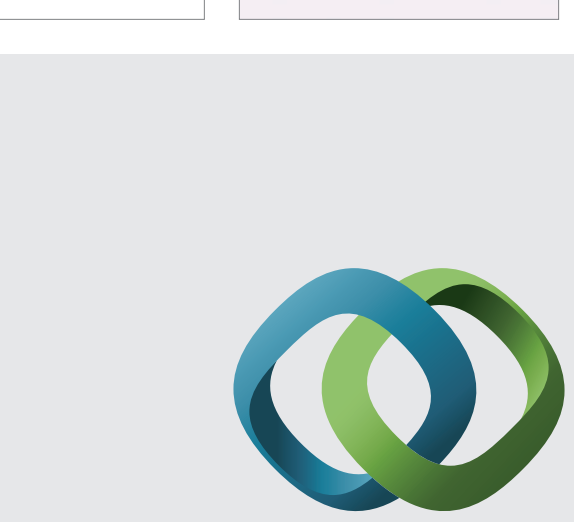

\section{Hindawi}

Submit your manuscripts at

http://www.hindawi.com
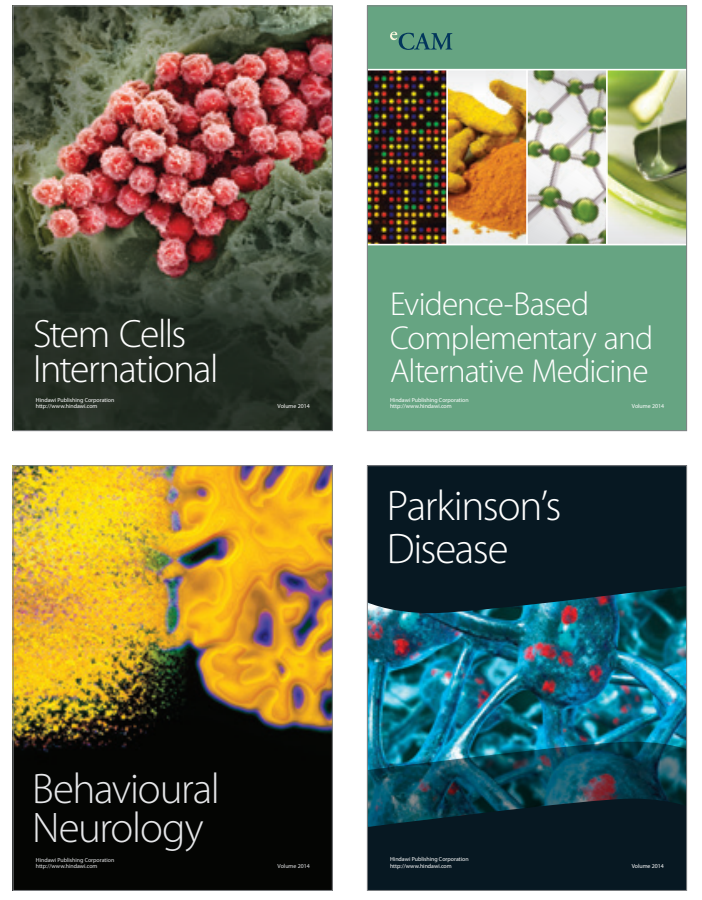
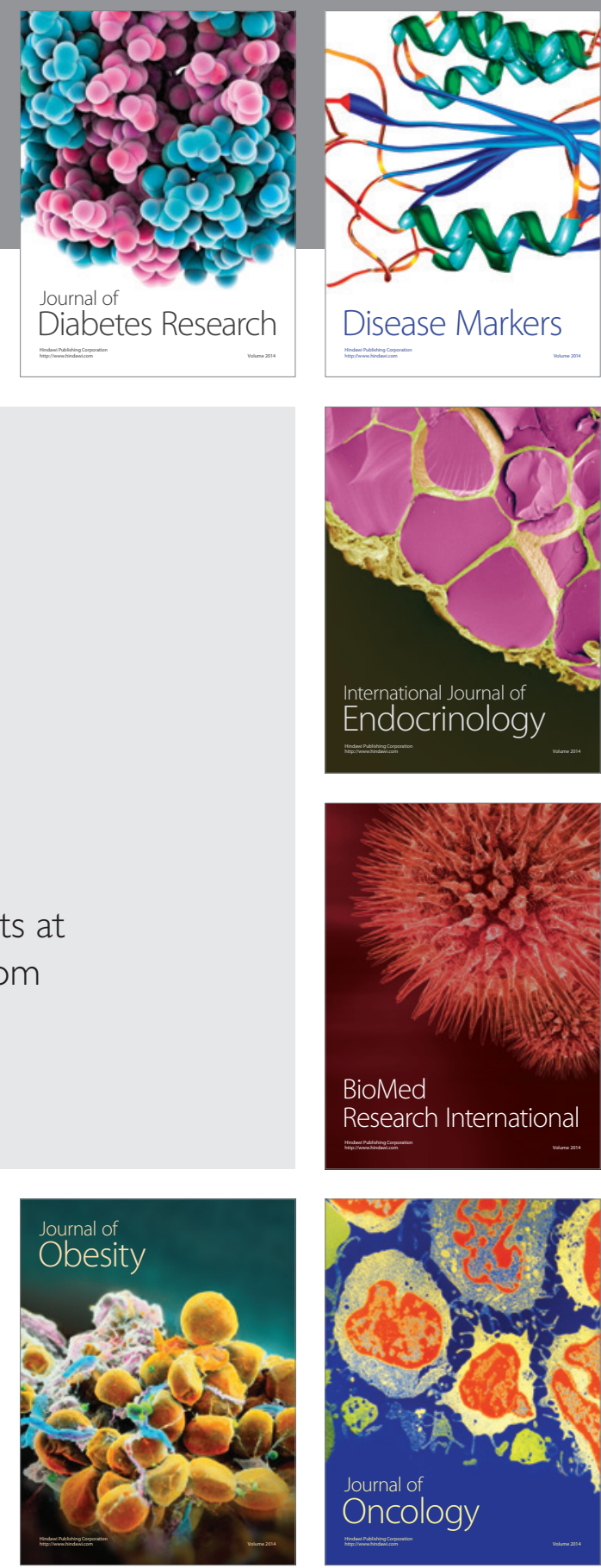

Disease Markers
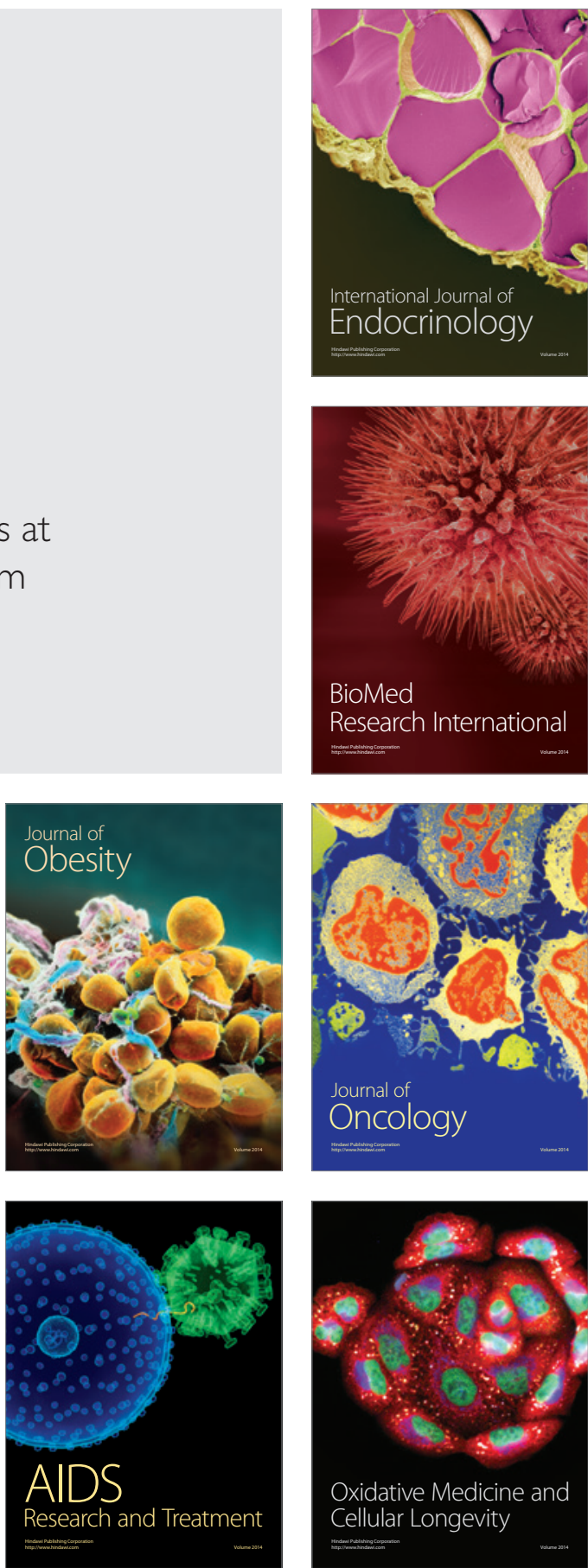